${ }^{0}$ Entomologica Fennica. 8 January 1998

\title{
Index to genera and species, Volume 8 (1997)
}

\section{Acari}

Camisia horrida (Herrmann) 181

Carabodes labyrinthicus (Michael) 181

Cymbaeremaeus cymba (Nicolet) 181

Eueremaeus oblongus C. L. Koch 181

Phauloppia coineaui Trave 181

- lucorum (C. L. Koch) 181

Phthiracarus sp. 181

Scheloribates latipes (C. L. Koch) 181

Zygoribatula exilis (Nicolet) 181

\section{Araneae \\ Agyneta innotabilis (O. P. Cambridge) 180 Anyphaena accentuata (Walckenaer) 180 Cheiracanthium oncognatum Thorell 180 Clubiona comta C. L. Koch 180 \\ Coleosoma floridatum Banks 7 Drapetisca socialis (Sundevall) 180 Hahnia pusilla C. L. Koch 180 Hypomma cornutum (Blackwall) 180, 183 Lepthyphantes minutus (Blackwall) 180 Micaria subopaca Westring 180 Moebelia penicillata (Westring) 180 Nuctenea umbratica (Clerck) 180 Salticus cingulatus (Panzer) 180 Savignya frontata (Blackwall) 180 Steatoda bipunctata (Linnaeus) 180 - grossa (C. L. Koch) 7 \\ Theridion pallens Blackwall 180 \\ - tinctum (Walckenaer) 180 \\ Triaeris stenaspis Simon 7 \\ Xysticus lanio C. L. Koch 180 \\ Zygiella atrica (C. L. Koch) 180 \\ - stroemi (Thorell) 180}

\section{Blattodea}

Ectobius sylvestris (Poda) 181

\section{Chilopoda}

Lithobius erythrocephalus C. L. Koch 180 - tenebrosus Meinert 180

\section{Coleoptera}

Absidia schoenherri (Dejean) 156, 158

Actobaena Gistel 68, 77

Agnoshydrus n. gen. 57, 59-60, 65-66, 68, 72-73

- densus n. sp. 57, 59, 64-65, 73-74

- laccophiloides (Régimbart) 57, 59, 61, 64-65, 73-74

Agonum (Agonum) dolens (C. Sablberg) 15

- (A.) impressum (Panzer) 15

- (A.) jankowskii Lafer 15

- (A.) sculptipes Bates 15

- (Eucolpodes) japonicum (Motschulsky) 15

- (Europhilus) gracile Sturm 15

- (E.) piceum (Linnaeus) 15

- (E.) subtruncatum Motschulsky 15

- (E.) thoreyi Dejean 15

- jankowskii Lafer 13

- piceum (Linnaeus) 13

- (Platynus) assimilis (Paykull) 15

Allopachria Zimmermann 57-61, 65-66, 68, 72, 74-76

- barong Hendrich \& Balke 76

- bimaculata (Satô) 59,62, 76

- flavomaculata (Kamiya) 59, 61, 76

(f. kumozuensis Satô) 76

- $\quad$ (f. narusei Satô) 76

- quadrimaculata (Satô) 76

- quadripustulata Zimmermann 59, 75-76

- sausai 61

- umbrosa Zimmermann 76

- wangi Nilsson \& Wewalka 59, 62-65, 76

Allophydrus Zimmermann 77

Amara spp. 24

- (Amara) communis (Panzer) 15

- (A.) similata chalcites Dejean 15

- (A.) ussuriensis Lutshnik 15

- (Zezea) plebeja (Gyllenhal) 15

Amischa decipiens (Sharp) 182

- nigrofusca (Stephens) 182

Ampedus balteatus (L.) 156

- erythrogonus (Müller) 156

- nigrinus (Hbst) 156

- nigroflavus (Goeze) 182

- pomorum (Hbst) 156

- tristis (L.) 156

Andex Sharp 57, 66, 68-69

- insignis Sharp 59-63, 68-69

Anisodactylus signatus (Panzer) 15

Anthaxia quadripunctata (L.) 156

Anthophagus omalinus Zett. 156 
Aplocnemus tarsalis (Sahlb.) 156

Apriophorus Guignot 77

Athous subfuscus (Müller) 156, 158

Aulacodytes Guignot 77

Bagous angustus (Silfverberg) 160

- cylindrus (Paykull) 160

- scanicus Silfverberg 160

Bembidion 13

- (Bembidion) paediscum Bates 14

- sp. pr. captivorum Netolitsky 14

- chloropus Bates 13

- (Chrysobracteon) conicolle Motschulsky 14

- (Cylindrobracteon) chloropus Bates 14

- (Diplocampa) prostratum Motschulsky 14

- (Eupetodromus) sibiricum Dejean 14

- grapeii 21

- inouyei Habu 14

- (Notaphus) obliquum fasciatum Motschulsky 14

- (Peryphus) dolorosum Motschulsky 14-15

- (P.) morawitzi Csiki 15

- (P.) ?pohlai Kirschenhofer 14

- (Plataphodes) tetraporum Bates 15

Bolitophagus 163

- reticulatus (L.) 161-165

Calathus micropterus 21-22, 24

Carabus (Adelocarabus) arboreus Lewis 14

- (Carabus) granulatus Linnaeus 14

- (Homoeocarabus) maeander Fischer von Waldheim 14

- violaceus 21

Cardiophorus ruficollis (L.) 156

Chlaenius (Chlaenius) insularis Kryzhanovskij 16

Chrysomelidae spp. 156

Cicindela (Cicindela) sachalinensis A. Morawitz 14

- (C.) sylvatica Linnaeus 14

- (C.) transbaicalica Motschulsky 14

Cis glabratus Mellie 161-165

- jacquemarti Mellie 161-165

- quadridens Mellie 161

Coelhydrus Sharp 57, 65-66, 68-69

- brevicollis Sharp 59-60,69

Coeliodes dryados (Gmelin) 182

Corticarina obfuscata Strand 156

Cryptophagus abietis (Payk.) 156

Curculio cylindrus Fabricius 160

Crypturgus cinereus (Hbst) 157

Cychrus caraboides 22, 24

Dalopius marginatus (L.) 156

Darwinhydrus Sharp 57, 66, 68-70

- solidus Sharp 59-60, 62, 69-70

Demetrias amurensis Motschulsky 16

Deronectes 58

Desmopachria Babington 57-61, 65-66, 68, 80-81

- (Desmopachria) Babington 66, 80-81

- (D.) aspera Young 81

- (D.) attenuata Régimbart 60, 80-81

- (D.) balfourbrownei Young 81

- (D.) bifasciata Zimmermann 81

- (D.) cenchramis Young 81
- (D.) circularis Sharp 81

- (D.) convexa (Aubé) 60, 80-81

- (D.) darlingtoni Young 81

- (D.) defloccata Young 81

- (D.) flavida Young 81

- (D.) geijskesi Young 81

- (D.) glabella Young 81

- (D.) glabricula Sharp 81

- (D.) grana (LeConte) 80-81

- (D.) granoides Young 81

- (D.) hylobates Young 81

- (D.) isthmia Young 81

- (D.) laesslei Young 81

- (D.) leechi Young 81

- (D.) lewisi Young 81

- (D.) liosomata Young 81

- (D.) majuscula Young 81

- (D.) (?) margarita Young 81

- (D.) nitida Babington 60, 80-81

- (D.) nitoides Young 81

- (D.) ovalis Sharp 81

- (D.) phacoides Guignot 81

- (D.) pulvis Guignot 81

- (D.) signata Zimmermann 81

- (D.) striga Young 81

- (D.) strigata Young 81

- (D.) subfasciata Young 81

- (D.) subnotata Zimmermann 81

- (D.) subtilis Sharp 81

- (D.) tarda Spangler 81

- (D.) voltata Young 81

- (D.) zelota Young 81

- (D.) zimmermani Young 81

- dispersa (Crotch) 61-62

- grouvellei Régimbart 80

- (Hintonella) Young 66, 80-81

- (H.) minuta (Young) 81

- (H.) siolii (Young) 81

- (H.) ubangoides (Young) 81

- (Hintonia) Young 80-81

_ iridis Young 80

- (Nectoserrula) Guignot 66, 80-81

- (N.) concolor Sharp 60, 80-81

- (N.) mendozana (Steinheil) 60-62, 64-65, 80-81

- (N.) punctatissima Zimmermann 60-61, 80-81

- (N.) vicina Sharp 60, 80-81

- nitida Babington 80

- (Pachiridis) Young 66, 80-81

- (P.) aldessa Young 81

- (P.) aureus Young 81

- (P.) iridis Young 60, 80-81

- (P.) novacula Young 81

- (Pachriodesma) Guignot 66, 80-81

- (P.) brevicollis Régimbart 60,80-81

- (P.) dispersa (Crotch) 80-81

- (P.) latissima (LeConte) 81

- (P.) mexicana Sharp 60-61, 80-81

- (P.) mutchleri Blatchley 81 
- (P.) sanfilippoi Guignot 60-61, 64-65, 80-81

- (P.) seminola Young 81

- (P.) suturalis Sharp 81

- (P.) varians Wehncke 81

- (Pachriostrix) Guignot 66, 80-81

- (P.) ferrugata Regimbart 81

- (P.) fossulata Zimmermann 81

- (P.) grouvellei Régimbart 60, 80-81

- (P.) paradoxa Zimmermann 81

- (P.) ruginosa Young 81

- (P.) striola Sharp 81

- (Portmannia) Young 66, 80-81

- (P.) basicollis Guignot 60, 80-81

- (P.) bryantstoni Clark 80-81

- (polita Sharp) 81

- (P.) decorosa Young 81

- (P.) dispar Sharp 60,81

- (P.) goias Young 81

- (P.) laevis Sharp 60, 80-81

- (P.) mutata Sharp 81

- (P.) niger Zimmermann 81

- (P.) nitidissima Zimmermann 81

- (P.) pittieri Young 81

- (P.) portmanni Clark 60-61, 63, 80-81

- (P.) sobrina Young 81

- (P.) speculum Sharp 81

- (P.) variegata Sharp 80-81

- (P.) zethus Young 81

- ubangoides Young 80

Diplous depressus Gebler 14

Dorcatoma 163-164

- chrysomelina Sturm 182

- punctulata Mulsant \& Rey 161-165

- robusta Strand 161-165

Dromius agilis (Fabricius) 156, 182

- fenestratus (F.) 156

- quadrimaculatus (Linnaeus) 182

- schneideri Crotch 156

Dytiscus ovatus Linnaeus 76

Elaphrus (Elaphrus) riparius (Linnaeus) 14

- (Neoelaphrus) sibiricus Motschulsky 14

Enicmus rugosus (Herbst) 182

Epuraea spp. 156

Euplectus kirbyi Denny 182

Gnathotrichus materiarius (Fitch) 153, 155, 157-158, 193195

- retusus 194

- sulcatus 194

Hadrobregmus pertinax (L.) 156

Hapalarea ioptera (Stephens) 182

Harpalus spp. 24

Heterhydrus Fairmaire 57-61, 63, 65-68

- adiaptus Guignot 67

- agaboides Fairmaire 67

- ghanensis Wewalka 58,67

- senegalensis (Aubé) 59,61-65, 67

- sudanensis Zimmermann 59, 62-63, 67

Hintonia Reichart 80
Hovahydrus Biström 57, 59-60, 65-66, 68, 79

- minutissimus (Régimbart) 59, 79

- perrieri (Fairmaire) 60, 79

- praetextus (Guignot) 60, 64-65, 79

- sinapi (Guignot) 60, 79

Hydrachna Fabricius 76-77

Hydrachna Müller 76-77

- gibba Fabricius 76

Hydroglyphus angularis 63

Hydropeplus Sharp 57, 66, 68, 70-71

- montanus Omer-Cooper 71

- trimaculatus (Laporte de Castelnau) 59-62, 64-65, 71

Hydroporus latissimus LeConte 80

- portmanni Clark 80

- trimaculatus Laporte de Castelnau 70

Hydrovatus Motschulsky 57

- laccophiloides Régimbart 72, 74

- maculatus Motschulsky 58

Hygrobia hermanni 76

Hygrotus Stephens 58, 80

Hylastes cunicularius Er. 157

Hylobius abietis (L.) 157

Hylurgops palliatus (Gyll.) 157

Hyphovatus Wewalka \& Biström 57, 66, 68, 71-72

- dismorphus (Biström) 59-60

- manfredi Wewalka \& Biström 72

- prapatensis Wewalka \& Biström 72

Hyphydrus Illiger 57, 59-60, 65-66, 68, 76-77

- abyssinicus Peschet 78

- aequatorialis Biström 79

- agnitus Guignot 77

- alfredi Bilardo \& Rocchi 77

- alluaudi Régimbart 77

- amplimaculatus Bilardo \& Rocchi 77

- anatolicus Guignot 78

- (carrarai Sanfilippo) 78

- assinicus Régimbart 77

- ater Bilardo 77

- aubei Ganglbauer 60, 64-65, 78

- (variegatus Aubé) 78

- barysomus Guignot 78

- bigamus Guignot 78

- birmanicus Régimbart 79

- bistroemi Bilardo \& Rocchi 77

- (hiekei Biström) 77

- boettcheri Biström 78

- brancuccii Biström 79

- burgeoni Gschwendtner 78

- caffer Boheman 77

- camerunensis Biström 78

- caryerus Guignot 78

- $\quad$ (guignoti Guéorguiev) 78

- (legrosi Guéorguiev) 78

- celebensis Biström 79

- celox Biström 79

- ceramensis Wewalka \& Biström 79

- circumflexus Klug 79

- (hardenbergi Zimmermann) 79 
— coccinelloides Zimmermann 78

- concii Bilardo \& Rocchi 79

- congoanus Biström 78

- conradsi Gschwendtner 79

- (opiparus Guignot) 79

- contiguus Wehncke 77

- cuppeni Biström 78

- cycloides Régimbart 78

- (circularis Régimbart) 78

- (lamottei Legros) 78

- (malawiensis Omer-Cooper) 78

- $\quad$ (nigeriensis Omer-Cooper) 78

- (pelates Guignot) 78

- dani Biström, Balke \& Hendrich 77

- decemmaculatus Wehncke 77

- (curvipes Régimbart) 77

- delibatus Guignot 78

- ? (brownei Bertrand) 78

- detectus Falkenström 78

- (chinensis Hlisnikovsky) 78

- (orrientalis v. reductus Hlisnikovsky) 78

- (pieli Guignot) 78

- dismorphus Biström 71

- dissimilis Biström 78

— distinctus Aubé 78

- ditylus Guignot 78

- effeminatus Watts 77

- eldenbecki Biström 79

- elegans (Montrouzier) 60-61, 63-65, 77

- (australis Clark) 77

- (bifasciatus Macleay) 77

- (blanchardii Clark) 77

- (caledoniae Clark) 77

- (gibbicollis Régimbart) 77

- (illigeri Perroud) 77

- (nitidicornis Broun) 77

- eremita Guignot 79

- esau Biström 79

- essoni Bilardo \& Rocchi 78

- excoffieri Régimbart 79

- facilis Biström 78

- falkenstromi Gschwendtner 78

- (brincki Guignot) 78

- fangensis Biström \& Satô 79

- flaviceps Zimmermann 77

- fluviatilis Pederzani 78

- funebris Guignot 77

- fuscus Omer-Cooper 77

- gabonicus Régimbart 78

- (vicinus Bertrand \& Legros) 78

- gibba Illiger 76

- gibbosus Biström 79

- grandis Laporte de Castelnau 60, 64-65, 77 (major Sharp) 77

- gschwendtneri Guignot 78

- (lindemannae Guignot) 78

- (pictus v. indicus Gschwendtner) 78

- (p. v. nalinie Vazirani) 78
- hermanni 76

- holmeni Biström 79

- holomelas Biström 79

— imitator Biström 79

— impressus Klug 79

- (cocquerelii Fairmaire) 79

- (flavicollis Régimbart) 79

- inopinatus Omer-Cooper 78

_ intermixtus (Walker) 79

- (indicus Sharp) 79

- j. japonicus Sharp 78

- (frontalis Sharp) 78

- (paromoeus Guignot) 78

- j. vagus Brinck 78

- jacobsoni Biström 79

- jaechi Wewalka \& Biström 79

- keiseri Mouchamps 78

- komghaensis Omer-Cooper 78

- l. flavicans Régimbart 77

- l. foveolatus Régimbart 77

- l. laeviventris Sharp 78

- l. lyratus Swartz 77

- (bisulcatus Clark) 77

- $\quad$ (fossulipennis Macleay) 77

- (nigronotatus Clark) 77

- l. tsugaru Nakane 78

- l. xanthomelas Régimbart 77

- lanzai Bilardo \& Rocchi 78

- lasiosternus Guignot 78

- (bertrandi Guéorguiev) 78

- lentiginosus Guignot 78

- linnavuorii Biström 77

- loriae Régimbart 79

- lyratus 65,76

- maculatus Babington 78

- (africanus Sharp) 78

- (a. v. borkuanus Bruneau de Miré \& Legros) 78

- (crassus Wollaston) 78

- maculiceps Régimbart 78

- (basilewskyi Guignot) 78

- maculifer Guignot 78

- madagascariensis Wehncke 77

- malkini Guignot 78

- mbandouensis Bilardo \& Rocchi 77

- mendozanus Steinheil 80

- microreticulus Bilardo \& Rocchi 77

- nasutus Bilardo \& Pederzani 79

- nigrovittatus Régimbart 78

- occultus Bilardo \& Rocchi 78

- odiosus Guignot 78

- omercooperae Guéorguiev 78

- opaculus Régimbart 78

- (caviceps Régimbart) 78

- orientalis Clark 77

- (eximius Clark) 78

- ovalis Illiger 76

- ovatus (Linnaeus) 60, 65, 77

- (ferrugineus Linnaeus) 77 
- (gibbus Fabricius) 77

- (grossus Müller) 77

- (ovalis Illiger) 78

- (sphaerius De Geer) 77

- (variegatus Stephens) 78

- parvicollis Sharp 60, 62-63, 77

- pavani Bilardo \& Rocchi 78

- pederzanii Biström 78

- perforatus Régimbart 78

- (p. v. alogus Guignot) 78

- (p. v. degener Guignot) 78

- (scitulus Omer-Cooper) 78

- pictus Klug 78

- $\quad$ (lugubris de Borre) 78

- praetextus Guignot 79

- prinzi Wewalka \& Biström 79

- prozeskyi Biström 79

- pulchellus Clark 78

- (jeanneli Guignot) 79

- (orbicularis Régimbart) 78

- (wui Gschwendtner) 78

- puncticollis Sharp 78

- quadriguttatus Guignot 78

- quadrisulcatus Bilardo \& Rocchi 78

- renardi Severin 79

- residuus Omer-Cooper 77

- sanctus Sharp 78

- satyrus Bilardo \& Pederzani 78

- schoedli Wewalka \& Biström 78

- schoutedeni Gschwendtner 77

- scripta Illiger 76

- scriptus (Fabricius) 78

- separandus Régimbart 60, 78

- (oncodes Guignot) 78

- signatus Sharp 78

- (aethiopicus Balfour-Browne) 78

- $\quad$ (grossus Sharp) 78

- silfverbergi Biström 78

- silvanus Bilardo 78

- sjoestedti Biström 78

- soni Biström 78

- spangleri Biström 79

- sphaeroidalis Biström 77

- stipator Guignot 78

- stipes Sharp 78

- $\quad$ (scriptus v. soarezicus Alluaud) 78

- subsignatus Bilardo \& Rocchi) 78

- sumatrae Régimbart 78

- (assamensis Vazirani) 78

- sylvester Guignot 78

- (spissus Guignot) 78

— tristiculus Guignot 77

- trophis Guignot 78

- tuberosus Guignot 78

- umilis Bilardo \& Rocchi 77

- variolosus Régimbart 78

- $\quad$ (v. v. patens Guignot) 78

- vasalloi Bilardo \& Rocchi 78
- villiersi Guignot 78

- wittei Gschwendtner 78

- zambiensis Pederzani 78

Ips 154-155, 157, 194

- acuminatus (Gyll.) 157

- duplicatus (Sahlb.) 153-159

- sexdentatus (Börner) 157

- typographus (L.) 153-159, 193

Laccornis 61

- oblongus (Stephens) 59-62

Leistus ferrugineus 21-22, 24

Leptusa ruficollis (Erichson) 182-183

Linaeidea aenea (L.) 156

Liotrichus affinis (Payk.) 156

Loricera pilicornis (Fabricius) 14, 21

Magdalis violacea (L.) 157

Megatoma undata (L.) 156

Methles 62

- cribratellus Fairmaire 59-61

Microdytes J. Balfour-Browne 57-61, 65-66, 68, 72, 74-75

- belli J. Balfour-Browne 59, 74-75

- championi J. Balfour-Browne 59, 75

- elgae Hendrich \& Balke 75

- laccophiloides (Régimbart) 74

- maculatus (Motschulsky) 75

- pasiricus (Csiki) 57, 59, 75

- (simplicior (Csiki)) 75

- (unicolor (Csiki)) 75

- sabitae Vazirani 75

- taiwanus Satô 75

— tomokunii Satô 75

— uenoi Satô 59, 75

Microlestes minutulus (Goeze) 13, 16

Mycetochara humeralis (Fabricius) 182

- obscura (Zett.) 156

Mycetophagus decempunctatus Fabricius 182

Nebria (Boreonebria) subdilatata Motschulsky 14

- (Reductonebria) ochotica R. F. Sahlberg 14

Nipponhydrus Guignot 76

Nipponhydrus Nilsson \& Wewalka 74-75

- flavomaculatus Kamiya 74

Notiophilus reiterii 22

Orthotomicus suturalis (Gyl1.) 157

Otiorhynchus scaber (L.) 156

- singularis (Linnaeus) 182

Pachyatheta mortuorum (Thomson) 182

Pachydrus Sharp 57-61, 63, 65-68

- brevis Sharp 68

- cayenennsis (Laporte de Castelnau) 59, 68

- cribratus Sharp 68

- globosus (Aubé) 59, 64-65, 68

- (punctatus Sharp) 68

- obesus Sharp 67-68

- obniger (Chevrolat) 68

- politus Sharp 68

- princeps (Blatchley) 59,68

- ritsemae Régimbart 67-68

Pachytes Montrouzier 77 
Paromalus flavicornis (Herbst) 182

Patrobus assimilis 19, 21-22, 24

-_ atrorufus 22

Phloeotribus spinulosus (Rey) 157

Pissodes gyllenhalii (Sahlb.) 157

- harcyniae (Hbst) 157

- pini (L.) 157

Pityogenes bidentatus (Hbst.) 157

- chalcographus (L.) 153-155, 157-158

Plectophloeus nitidus (Fairmaire) 182-183

Plegaderus caesus (Herbst) 182

Poecilus (Macropoecilus) fortipes Chaudoir 15

Polydrusus pilosus Gredler 156

- undatus (F.) 157

Primospes Sharp 57, 66, 68, 71

- suturalis Sharp 59-60, 62, 71

Prionychus ater (Fabricius) 182

Pterostichus ssp. 13, 24

- aethiops Panzer 16

- (Argutor) diligens (Sturm) 15

- (Badistrinus) neglectus A. Morawitz 15

- (Bothriopterus) adstrictus Eschscholz 15

- crassiceps A. Morawitz 13, 16

- (Cryobius) kurasawai Tanaka 15

- (Euryperis) Motschulsky 16

- (Euryperis) eximius A. Morawitz 13, 15-16

- (Eurythoracana) haptoderoides Tschitscherine 15

- eximius A. Morawitz 16

- (Melanius) nigrita (Paykull) 15

- neglectus A. Morawitz 13

- obliquebasalis Jedlicka 13, 16

- prochazkorum Jedlicka 13, 16

- rudnicus Jedlicka 13, 16

- sakhalinensis Kirschenhofer 13, 16

- (Steropus) Stephens sensu auct. 16

- (Steropus) alacer A. Morawitz 15

- (S.) crassiceps 16

- (S.) sakhalinensis 16

Ptinus subpilosus Sturm 182

Queda Sharp 57

Quedius brevicornis Thomson 182

Rhagium inquisitor (L.) 156

Salpingus planirostis (F.) 156

Scolytus intricatus (Ratzeburg) 182

Scraptia fuscula Müller 182

Sericus brunneus (L.) 156

Stenolophus (Stenolophus) propinquus A. Morawitz 15

Steropus 16

Strophosoma capitatum (Degeer) 157

Syneta betulae (F.) 156

Tetropium castaneum (L.) 156

Thamiaraea hospita (Märkel) 182

Thanasimus 153,157

- femoralis (Zett.) 154-158

- formicarius (L.) 154-158

Trypodendron lineatum (Oliv.) 157, 194

Xyletinus pectinatus (Fabricius) 182

\section{Diplopoda}

Cylindroiulus britannicus (Verhoeff) 180

Nemasoma varicorne C. L. Koch 180

Polyxenus lagurus (Linnaeus) 180

Proteroiulus fuscus (Am Stein) 180

\section{Diptera}

Camptochironomus Kieffer 215-218

?Camptolabis 218

Chaetolabis Townes 215

Chironomus Meigen 171, 173, 215, 217-218

sp. "Seitajärvi" 39, 45-48, 51-53

- aberratus 50-53

- aberratus Keyl 39-40, 50-51, 172

- anthracinus Zetterstedt 51, 171-172, 174

- balatonicus 174

- beljaninae Wülker 50-52, 172-174

- (Camptochironomus) sp. 217

- (C.) pallidivittatus auctt. 215-218

- (C.) tentans (Fabricius) 215-218

- esai Wülker 171-176

- fraternus Wülker 39, 50-53, 172-174

- holomelas 172

- jonmartini Lindeberg 39-55, 172

- major 172, 174

- melanotus Keyl 51

- muratensis 174

- neglectus 39

- neocorax 172

- obtusidens 51

- piger 173

- pilicornis 172

- plumosus Linnaeus sensu auctt. 40, 173, 218

- riihimakiensis Wülker 51, 172, 216, 218

- salinarius 51

- semireductus 171, 173-174

- sororius Wülker 40, 50-51

- staegeri 51-52, 172

- staegeri Lundbeck 52

- tardus 172

- tentans 216

- var. pallidivittatus Malloch 216

- tenuistylus Brundin 51, 172, 174

- whitseli 172

Epistrophe cryptica Dozckal \& Schmid 2

Epistrophella euchroma (Kowartz) 2

Eupeodes abiscoensis (Dusek \& Laska) 2-3

- borealis (Dusek \& Laska) 2

- latilunulatus (Collin) 2

Helicophagella melanura (Meigen) 3

Lobochironomus 215, 218

Lydella ripae Brischke 2

Microtendipes lugubris Kieffer 54

- nigellus Hirvenoja 54 
Platycheirus amplus Curran 1

- nielseni Vockeroth 1-2

- occultus Goeldlin, Maibach \& Speight 2

Procladius sp. 54

Procladius breviatus Remmert 54

Protophormia terraenovae (Robineau-Desvoidy) 3

Sciopus contristans (Wiedemann) 1

- maritimus Becker 1

Sphaerophoria bankowskae Goeldlin 3

Tipula 215

\section{Heteroptera}

Aquarius adelaidis (Dohrn) 167

Callicorixa producta 53

Calocoris ochromelas (Gmelin) 181

Cyllecoris histrionicus (Linnaeus) 182

Dryophilocoris flavoquadrimaculatus (De Geer) 182

Empicoris vagabundus (Linnaeus) 181

Gerris argentatus Schumm. 186-188

- lateralis Schumm. 186-188, 190

- odontogaster (Zett.) 186-189

- spinolae Lethierry \& Severin 167

- thoracicus Schumm. 186-190

Halobates spp. 186

Limnogonus franciscanus (Stål) 167-170

Loricula pselaphiformis Curtis 181

Myrmedobia distinguenda Reuter 181, 183

Phylus melanocephalus (Linnaeus) 182

Pilophorus perplexus (Douglas \& Scott) 181-182

Psallus mollis (Mulsant \& Rey) 182

- perrisi (Mulsant \& Rey) 182

- variablis (Fallén) 182

- wagneri Ossiannilsson 182

Temnostethus gracilis (Horvath) 181

\section{Homoptera}

Alnetoidia alneti (Dahlbom) 180-181

Edwardsiana frustrator (Edwards) 181

Eurhadina concinna (Germar) 181

- pulchella (Fallén) 181

Iassus lanio (Linnaeus) 181

Lachnus roboris (Linnaeus) 181

Phylloxera coccinea von Heyden 181

Thelaxeres dryophila (Schrank) 181

Trioza remota Förster 181

Tuberculatus annulatus (Hartig) 181

\section{Hymenoptera}

Andrena 109-110, 208

- clarkella (Kirby) 207-208, 21.1-212

- denticulata (Kirby) 209, 212
- fulvago (Christ) 209

- fuscipes (Kirby) 208, 210

- gelriae van der Vecht 210

- hattorfiana 205, 207, 209, 21 I-212

- humilis Imhoff 209

- intermedia Thomson 210, 212

- labialis (Kirby) 210

- lapponica 207, 210

- lathyri Alfken 207, 210

- marginata 207, 209

- nanula Nylander 209

- praecox (Scopoli) 211-212

- ruficrus Nylander 207-208, 211-212

- tarsata Nylander 207, 210

- vaga Panzer 109-110, 211

- wilkella Kirby 210,212

Aneugmenus spp. 35

- fuerstenbergensis (Konow) 37

Anthidium 208

Anthocopa 208

Anthophora 208

- furcata (Panzer) 210, 212

Birka Malaise 38

- cinereipes (Klug) 27-38

Bombus 208, 212

- consobrinus Dahlbom 205, 208, 210

Ceratina 208

Chalicodoma 208

- ericetorum Lepeletier 210

Chelostoma 207-208

- campanularum (Kirby) 209

- florisomne (L.) 208, 210

- maxillosum (L.) 210

- rapunculi (Lepeletier) 209

Colletes 208

- cunicularius (L.) 211-212

- daviesanus F. Smith 208-209, 211

- similis Schenck 209

- succinctus (L.) 208, 210

Dasypoda 208

- altercator (Harris) 209

- hirtipes (Latreille) 209

Dolerus gonager (Fabricius) 37-38

Dufourea 208

- dentiventris (Nylander) 209, 212

- inermis (Nylander) 209

- minuta Lepeletier 208

- vulgaris (Schenck) 209

Eriocampidea 37

Eucera 208

- longicornis (L.) 205, 207, 210-212

Euura Newman 114

- amerinae L. 114, 117-118

- atra (Jurine) 113-119

- exiguae Smith 114

- lasiolepis Smith 114

- mucronata (Hartig) 114-115

- "mucronata" 114

- s-nodus? Walsh 114 
Formica fusca Linnaeus 182

- polyctena Förster 180, 182

- rufa Linnaeus 182

Halictus 207-208

Heriades 208

- truncorum (L.) 209

Hoplitis 208

- mitis Nylander 208

Hylaeus 208

- nigritus (Fabricius) 209

- signatus (Panzer) 208, 210

Ichneumon coerulator Fabricius 5

Lasioglossum 207-208

Lasius mixtus (Nylander) 182

- niger (Linnaeus) 182

Macropis 207-208, 211-212

- europaea Wamcke 210

- fulvipes (Fabricius) 210

- labiata auct. 210

Megachile 208

- bombycina Radoszkowski 209

- lapponica Thomson 207-208, 210

- nigriventris Schenck 210

Melecta 208

Melisandra Benson 38

- cinereipes 32,37

Melitta 208

- haemorrhoidalis (Fabricius) 209

- leporina (Panzer) 210

- tricincta Kirby 208

Myrmica ruginodis Nylander 182

Nesoselandria Rohwer 27, 37-38

- morio (Fabricius) 27-38

Osmia 208

- leaiana (Kirby) 209

Panurginus 208

Panurgus 208

- banksianus (Kirby) 208

- calcaratus (Scopoli) 208-209

Periclista lineolata (Klug) 38

Pimpla spp. 5

Platygaster 203

- americana (Ashmead) 203

- attenuata 203

- betularia Kieffer 202

- entwistlei Buhl 202

- litoralis sp. n. 197, 200-202

- nitida 203

- phragmitis 203

- rohweri Fouts 202

Prosactogaster 203

- attenuata (Haliday) 202

- damokles sp. n. 197, 202-203

- enneatomus (Kieffer) 202

- lineata (Kieffer) 202

- marshalli Kieffer 202

- nitida (Thomson) 202

- phragmitis (Schrank) 202
- subuliformis Kieffer 202

Rocalia 37

- longipennis Takeuchi 37

Rophites 208

Selandria aperta 32

Strongylogaster macula (Klug) 37

- mixta (Klug) 37

Synopeas 198-199

- affinis (Nees) 199

- bohemani sp. n. 197, 199-200

- brevis sp. n. 197-199

- trebius (Walker) 199

- velutinus (Walker) 199-200

Telenomus albatorius Kozlov \& Kononova 198

- ciliatus sp. n. 197-198

- cubiceps Ashmead 198

- heteropterus Haliday 198

- microceps Szabó 198

- minimus Kozlov 198

- zethos Walker 198

Trachysa 208

- bysina (Panzer) 210, 212

Trigona 205

Trogus Panzer 5

- lapidator 5

- lapidator coerulator (Fabricius) 5

- l. lapidator (Fabricius) 5

- l. panzeri Carlson 5-6

\section{Lepidoptera}

Abraxas sylvatus (Sco.) 128

Acasis viretata (Hb.) 131

Acherontia atropos (L.) 123, 131

Acleris ferrugana (Denis \& Schiffermüller) 182

Acontia lucida (Hfn.) 132

Acronicta euphorbiae (D. \& S.) 131

- leporina (L.) 131

- rumicis (L.) 131

- strigosa (D. \& S.) 128,139

Aethes tesserana (D. \& S.) 134, 137

Aglais urticae 134

Agonopterix laterella (D. \& S.) 137

Agriopis 126

- aurantiaria (Hb.) 131, 134-135, 139

- marginaria (F.) 132

Agrius convolvuli (Linnaeus) 123-124

Agrochola macilentus (Hb.) 133, 135-136

Agrotis cinerea (D. \& S.) 132

— ipsilon (Hufnagel) 124-125

- segetum (D. \& S.) 122

Alcis jubatus (Thb.) 123, 129, 136

Allophyes oxyacanthae (L.) 128

Ammoconia caecimacula (D. \& S.) 125,128

Amphipyra 182

- berbera Rungs $123,130,182$

- livida (D. \& S.) 132 
- perflua (Fabricius) 123, 130, 133-134, 136, 139, 182

- pyramidea (L.) 130

- tragopoginis (Clerck) 134-135

Anticlea derivata (D. \& S.) 131, 134

Apamea Ochsenheimer 83, 85

- ferrago (Eversmann) 83, 85

- lithoxylaea (D. \& S.) 133

- nekrasovi Mikkola, Varga \& Guylai 83, 85

- ophiogramma (Esper) 125, 129, 135

- scolopacina (Esp.) 130, 132-133, 135-136, 139

- sordens (Hfn.) 131

- yunnana Kononenko \& Mikkola 83-85

- zollikoferi (Freyer) 125

Apatura iris 140

Aplocera praeformata (Hb.) 128-129, 135

Apocheima 126

_ pilosaria (D. \& S.) 131

Aporia crataegi (Linnaeus) 123

Aporophyla lutulenta (D. \& S.) 132

Araschnia levana (L.) 132, 134, 136, 140

Archanara dissoluta (Tr.) 125, 129

- geminipuncta (Hw.) 132-133

- sparganii (Esp.) 125, 128

Arenostola semicana (Esp.) 128

Argynnis paphia (L.) 129

Atolmis rubricollis (L.) 129

Atypha pulmonaris (Esp.) 132

Autographa buraetica (Staudinger) 123

- excelsa (Kretschmar) 129

- gamma (Linnaeus) 123-124

- macrogamma (Eversmann) 134, 136

- mandarina (Fr.) 131-132, 135-136, 139

Axylia putris (L.) 128

Baptria tibiale (Esp.) 131, 141

Blepharita amica (Treitschke) 134-135

- satura (D. \& S.) 128

Calamia tridens (Hfn.) 125, 128-129, 139

Callimorpha dominula (Linnaeus) 123

Calliteara pudibunda (L.) 129

Caloptilia hemidactylella (D. \& S.) 137

Campaea margaritata (Linnaeus) 133, 135, 139

Catarhoe rubidata (D. \& S.) 125, 128, 134

Catascythris kebirella Amsel 89

Catocala 130

- adultera (Ménétriés) 125

- nupta (Linnaeus) 126

- pacta (L.) 131

Celaena leucostigma (Hb.) 128

Cepphis advenaria (Hb.) 128, 136

Cerastis leucographa (D. \& S.) 131, 134

Chersotis andereggii (Boisduval) 125

- a. arcana Mikkola 133

Chesias legatella (D. \& S.) 132

Chloroclysta infuscata (Tengström) 134

- siterata (Hfn.) 131, 135

Chloroclystis v-ata 133, 135-136

- v. relicta (Haworth) 133

- v. v-ata 138
Chortodes brevilinea (Fenn) 132

- elymi (Treitschke) 2

Cidaria fulvata (Forst.) 131, 136, 139

Cilix glaucatus (Sco.) 132

Cleorodes lichenarius (Hufnagel) 123

Clossiana freija (Thb.) 131

Cochylis hybridella (Hübner) 134, 137

Coenonympha glycerion (Br.) 128

- hero (L.) 132

Coleophora 9, 11

- badiipennella (Duponchel) 137

- baltica (Toll.) 9

- betulaenanae Klimesh 145, 149

- canadensisella McDunnough 146

- carelica Hackman 9-12

- expressella Klemensiewicz 11

- graminicolella (Heinemann \& Wocke) 9

- limosipennella (Duponchel) 137

- silenella var. graminicolella 9

- vacciniella H.-S. 145-150

- f. pallescentella Kanerva 148

Colias hyale (Linnaeus) 123

Colobochyla salicalis (D. \& S.) 128

Colotois 126

- pennaria (L.) 129, 139

Comibaena bajularia (D. \& S.) 132

Conistra erythrocephala (D. \& S.) 133

Cornutiplusia circumflexa (L.) 132

Cosmia affinis (L.) 132

- pyralina (Denis \& Schiffermüller) 3

- trapezina (L.) 131

Costaconvexa polygrammata (Br.) 132

Cryphia algae (F.) 132

- ereptricula (Tr.) 132

Cryptocala chardinyi (Bdv.) 130, 139

Cucullia absinthii (Linnaeus) 123

- boryphora Fsch. de W. 130, 132

- fraterna Butl. 130, 132

- verbasci (Linnaeus) 123

Cyclophora pendularia (C1.) 128-129, 140

Cydia discretana (Wocke) 137

- lunulana(D. \& S.) 137

Daphnis nerii (Linnaeus) 123

Deileptenia ribeata (Clerck) 128-129, 139

Deltote bankiana (F.) 132

Depressaria emeritella Stainton 137

Diarsia brunnea (D. \& S.) 125, 128

Diasemia reticularis (Linnaeus) 137

Discoloxia blomeri (Curt.) 131-133, 136

Drepana binaria (Hfn.) 132

Ectoedemia intimella (Zeller) 103

Eilema complanum (L.) 128

- depressum (Esp.) 129

- sororculum (Hfn.) 132

Elachista 219-223

- argentella 219

- aurocristata Braun 223

- bifasciella 219-220 
- eskoi 152

- jaskai sp. n. 219-221

- orestella (Busck) 223

- ripula sp. n. 219, 221-223

- triatomea Haworth 219, 222-223

Elaphria venustula (Hb.) 132

Endrosis sarcitrella (Linnaeus) 137

Ennomos alniarius (Linnaeus) 134

- autumnarius (Wern.) 131, 134, 136

Entephria polata (Duponchel) 87-88

Epirrhoe rivata (Hb.) 132

__ tartuensis Möls 132

Erannis 126

- defoliaria (Cl.) 131

Erebia embla (Thb.) 131

Eremobina pabulatricula (Bra.) 131

Erigethes strobilacei Walsingham 89

Eriocrania subpurpurella (Haworth) 182

Eublemma purpurinum (D. \& S.) 130, 132

Euchalcia modestoides (Poole) 132-133

- variabilis (Pil. \& Mit.) 132

Euchloe chloridice (Hb.) 130, 132

Eucosma campoliliana (D. \& S.) 137

Eugnorisma glareosum (Esp.) 130, 132

Eugraphe sigma (D. \& S.) 125

Eulithis pyropata (Hübner) 129, 135

Euphydryas editha 141

Eupithecia cauchiata (Dup.) 132

- irriguata (Hb.) 132

- linariata (D. \& S.) 128

- pernotata Guenée 126

- p. enictaia Pellmyr \& Mikkola 133

- sinuosaria Ev. 128, 135

Euxoa ochrogaster (Guenée) 125

- recussa (Hb.) 131

Everes alcetas (Hoff.) 132

Fixsenia pruni (L.) 129

Glaucopsyche alexis (Poda) 131, 139

Gortyna flavago (D. \& S.) 125,128

Gymnoscelis rufifasciata (Hw.) 128-129, 135

Gynaephora selenitica (Esp.) 129, 141

Gypsonoma oppressana (Treitschke) 137

Habrosyne pyritoides (Hfn.) 132-133, 135-136

Hadena irregularis (Hfn.) 132

- luteago (D. \& S.) 132

Hellinsia distincta (Herrich-Schäffer) 3

Helicoverpa armigera ( $\mathrm{Hb}) 130,$.

Heliothis nubigera $\mathrm{Hr}$.-Sch. 130, 132

Hemithea aestivaria (Hb.) 128, 134

Hepialus humuli (Linnaeus) 136-137

- lupulinus (Linnaeus) 137

Herminia grisealis (D. \& S.) 125,128

- tarsicrinalis (Knoch) 132

- tarsipennalis Tr. 128, 134

- tristalis 134

Hillia iris (Zetterstedt) 135

Hoplodrina ambigua 132

Horisme aemulata (Hb.) 132
- vitalbata (D. \& S.) 130,132

Hyles euphorbiae (Linnaeus) 123

- lineata (Esp.) 123, 130, 132

- lineata (Fabricius) 123, 130

Hypodryas maturna (L.) 131

Hypomecis punctinalis (Sco.) 128

- roboraria (D. \& S.) 128-129

Idaea biselata (Hfn.) 128

- dimidiata (Hfn.) 128

Inachis io (Linnaeus) 123, 126

Iphiclides podalirius (Sco.) 130, 132, 134

Ipimorpha contusa (Fr.) 132

Issoria lathonia (L.) 123, 131, 139

Klimeschiopsis kiningerella (Duponchel) 182

Lacanobia splendens (Hb.) 132, 135

Lamprotes c-aureum (Knoch) 125

Laothoe amurensis (Stgr.) 128-129

Larentia clavaria (Hw.) 128

Lasionycta skraelingia (Hr.-Sch.) 132-133

Laspeyria flexula (D. \& S.) 129

Leucania comma (L.) 131

Limenitis populi (L.) 122, 131

Lithophane ornitopus (Hufnagel) 133, 135

- semibrunnea (Hw.) 130, 132

Lobesia abscisana Doubleday 137

Lomographa bimaculata (F.) 128-129, 136

- temerata (D. \& S.) 128

Lopinga achine 123, 129

Lycaena dispar (Hw.) 130, 132, 134

- helle (D. \& S.) 131

- hippothoe (L.) 131

Macdunnoughia confusa (St.) 125, 128, 136

Macrochilo cribrumalis (Hb.) 131

Macroglossum stellatarum (Linnaeus) 123

Malacosoma neustria (Linnaeus) 3

Mamestra brassicae (L.) 131

Maniola jurtina (L.) 123, 128, 139

Meganephria oxyacanthae 139

Melanchra persicariae (L.) 128

Mesoligia furuncula (D. \& S.) 128

- literosa (Hw.) 130, 135

Millochrista miniata (Forst.) 129

Moma alpium (Osb.) 131

Mompha divisella Herrich-Schäffer 137

Mormo maura (Linnaeus) 132

Mythimna albipuncta (D. \& S.) 132

Narraga fasciolaria (Hfn.) 132

Nemophora cupriacella (Hübner) 137

Noctua interjecta Hübner 132

- interposita (Hb.) 132

- janthe (Br.) 132

- janthina (D. \& S.) 132

- pronuba (L.) 123, 125, 129

Nola karelica Teng. 131, 134

Nomophila noctuella (D. \& S.) 124

Nymphalis polychloros (Linnaeus) 123

Odontosia sieversi (Mén.) 128-129, 136, 139

Opigena polygona (D. \& S.) $131,134,139$ 
Orthosia cerasi (F.) 131, 133

- cruda 133

- gracilis (D.\& S.) 128, 141

- miniosa (D. \& S.) 132,135

- munda(D. \& S.) $130,132,135$

- populeti (F.) 131

Ourapteryx sambucaria (L.) 123, 131-133, 139

Pachetra sagittigera (Hufnagel) 125

Pammene argyrana (Hübner) 182

- splendidulana (Guenée) 182

Papilio Linnaeus 5

- machaon Linnaeus 5-6

Paradarisa consonaria (Hb.) 133, 136

Pararge achine (Sco.) 128

Parnassius apollo (L.) 131

- mnemosyne Linnaeus 224

Peribatodes rhomboidaria (D. \& S.) 132

- secundarius (D. \& S.) 132-133, 135-136, 139

Peridroma saucia (Hb.) 132

Perizoma affinitatum (St.) 131

- $\operatorname{sagittatum}(\mathrm{F}) 131,$.

Phalaeana celsia 124

Phragmatiphila nexa (Hb.) 132

Pieris brassicae (Linnaeus) 123

- rapae (Linnaeus) 123

Plagodis dolabraria (L.) 128

Polia conspicua (A. B.-H.) 132-133

- nebulosa (Hfn.) 128

Polypogon lunalis (Sco.) 128, 134, 141

Prodotis stolida (F.) 125, 130, 132

Protexarnis squalida (Gn.) 130, 132

Protodeltote pygarga (Hfn.) 128

Pseudohadena immunda (Eversmann) 125

Psoricoptera gibbosella (Zeller) 182

Ptilophora plumigera (D. \& S.) 130, 132

Pyralis lienigialis (Zeller) 137

Pyrrhia umbra (Hfn.) 129

Quercusia quercus (Linnaeus) 133

Rhizedra lutosa (Hb.) 131

Rhyparia purpurata (L.) 123, 129, 134

Rivula sericealis (Sco.) 131

Schinia scutosa (Denis \& Schiffermüller) 3, 123

Schrankia costaestrigalis (St.) 128

Scopula decorata (D. \& S.) 134

Scythris ammobia Falkovitch 90-91

- amphonycella (Geyer) 90-91,93

- andersi Bengtsson 95

- baikalensis Bengtsson \& Liška 90, 93

- bifissella (Hofmann) 90-91, 100-101

- braschiella (Hofmann) 95

- canescens (Staudinger) 92

- capitalis (Erschoff) 92-93

- cassiterella (Snellen) 92,99

- chrysopygella Caradja 99

- cuspidella (Denis \& Schiffermüiller) 90, 94

- fallacella (Schläger) 92

- felesella Bengtsson 89,92-93,96

- flavilaterella (Fuchs) 90, 92, 94, 99
- fuscopterella Bengtsson 94

- inspersella (Hübner) 94

- jalavai Sinev 94

— kailai Bengtsson 89,94-96

- kullbergi Bengtsson 89, 95-96

- kyzylensis Bengtsson 89, 94-95, 97

- laminella (Denis \& Schiffermüller) 94, 97

- langohri Passerin d'E \& Vives 95

- monochreella (Ragonot) 98

- mus Walsingham 98

- nitidella Bengtsson \& Liška 98, 100

- nivicolor Meyrick 98

- obscurella (Scopoli) 90

- pascuella (Zeller) 91

- paullella (Herrich-Schäffer) 91

- picaepennis (Haworth) 98

- pudorinella (Möschler) 91

- sinensis (Felder \& Rogenhofer) 98

- subcassiterella Bengtsson 89, 98-100

- terekholensis Bengtsson 89, 99-102

- tessulatella Rebel 102

- tityrella Falkovitch 99

Scythropia crataegella (Linnaeus) 137

Sedina buettneri (Her.) 132

Selidosema plumarium (D. \& S.) 132

Senta flammea (Curtis) 125

Shargacucullia sp. nr. lychnitis 132

Sideridis albicolon (Hb.) 130, 132

Spaelotis clandestina (Har.) 123, 131

— ravida (D. \& S.) 131

Spilosoma luteum (Hfn.) 128-129

Staurophora celsia (L.) 124, 128-129

Stauropus fagi (L.) 123, 128

Stenolechia gemmella (Linnaeus) 182

Stigmella 104

- benanderella (Wolff) 103

- lappovimella (Svensson) 103-107

- obliquella (Heinemann) 103

- salicis (Stainton) 103-104

- vimineticola (Frey) 107

- zelleriella Snellen 103-104, 106-107

Syndemis histrionana (Frölich) 137

Syngrapha ain (Hoch.) 130, 132

Tholera cespitis (D. \& S.) 125, 128

- decimalis (Poda) 125, 128

Tischeria ekebladella (Bjerkander) 182

Tortrix viridana (Linnaeus) 182

Trachea atriplicis (L.) 129, 134

Trichophaga tapetzella (Linnaeus) 137

Trichoplusia ni (Hb.) 132

Trichosea ludifica (Linnaeus) 124

Trisateles emortualis (D. \& S.) 128

Tyria jacobaeae (L.) 123, 130, 132

Vanessa atalanta (Linnaeus) 123

- cardui (Linnaeus) 123

Venusia cambrica Curt. 131, 134

Xanthia aurago (D. \& S.) 123, 132-133, 135

- gilvago (Denis \& Schiffermüller) 3, 132, 135 
Xestia 126

- brunneopicta (Mats.) 132-133

- collina (Bdv.) 131-132

- sexstrigata (Hw.) 125, 128-129, 134

- sincera (Herrich-Schäffer) 123

- xanthographa (D. \& S.) 131

Xylomoia strix Mikk. 130, 132

Ypsolopha chazariella (Mann) 137

- sylvella (Linnaeus) 182

- ustella (Clerck) 182

Zeiraphera isertana (Fabricius) 182

\section{Neuroptera}

Coniopteryx borealis Tjeder 181

\section{Opiliones}

Leiobunum rupestre (Herbst) 180 Mitopus morio (Fabricius) 180 Nelima gothica Lohmander 180 Rilaena triangularis (Herbst) 180

\section{Pseudoscorpionida}

Chernes cimicoides (Fabricius) 180

\section{Psocoptera}

Amphigerontia intermedia (Tetens) 1

Graphopsocus cruciatus (Linnaeus) 181

Lachesilla quercus (Kolbe) 181

Loensia fasciata (Fabricius) 181

- pearmani Kimmins 181

- variegata (Latreille) 181

Pseudopsocus fusciceps (Reuter) 181, 183

Reuterella helvimacula (Enderlein) 181

Trichadenotecnum incognitum Roesler 181

- majus (Kolbe) 181

\section{Strepsiptera}

Stylops 109-111

— melittae Kirby 109

- pacifica Bohart 109 